Z. klin. Chem. u. klin. Biochem.

9. Jg., S. 419-420, September 1971

\title{
Dünnschichtchromatographische Untersuchung von Phenolrot
}

\author{
Von B. Marowski und W. Fabricius
}

\author{
Aus dem Institut fïr Klinische Chemie und Klinische Biochemie (Direktor: Prof. Dr. H.-J. Dulce) im Klinikum Steglitz \\ der Freien Universität Berlin
}

(Eingegangen am 28. August 1970/15. Juni 1971)

\begin{abstract}
Der zur Nierendiagnostik verwendete Farbstoff Phenolsulfonphthalein (Phenolrot) wurde mit Hilfe der Dünnschichtchromatographie auf seine Reinheit untersucht. Sowohl die kommerziellen Originallösungen als auch selbst angesetzte wäßrige Lösungen der Reinsubstanz ergaben bei der Dünnschichtchromatographie mehrere Fraktionen. Mögliche Konsequenzen, die bei der Verwendung von in ihrer Einheitlichkeit nicht streng definierbaren Phenolrotlösungen in der Nierenfunktionsdiagnostik bedacht werden sollten, werden diskutiert.
\end{abstract}

\section{Thin layer cbromatographic study of phenol red}

The purity of phenolsulphonphthalein (phenol red), which is used in kidney diagnosis, was investigated by thin layer chromatography. Both the commercial solutions and the aqueous solutions of the pure substance prepared in the laboratory showed several fractions in thin layer chromatography. The possible consequences are discussed of using phenol red solutions whose purity cannot be strictly defined in the diagnosis of kidney function.

Obwohl das Phenolrot seit nahezu 60 Jahren in der Nierendiagnostik Anwendung findet $(1-3)$, konnte bisher die Frage, ob sich der Farbstoff metabolisch inert verhält, nicht geklärt werden. Bei Bilanzversuchen war immer wieder aufgefallen, daß etwa $20 \%$ einer intravenös injizierten Dosis von $6 \mathrm{mg}$ Phenolrot nicht. mehr sicher auffindbar waren (5). Dieser Verlust wird auch nicht durch den geringen Prozentsatz Phenolrot erklärt, der beim Menschen in die Galle ausgeschieden wird, und der nach MCLEOD und Mitarbeitern (5) ungefähr $3 \%$ einer intravenös injizierten Dosis von 5 mg entspricht.

Bei der Frage nach dem metabolischen Verhalten von Phenolrot und dem Verbleib des bislang nicht auffindbaren Farbstoffanteils von $20 \%$ muß zuerst die Einheitlichkeit handelsüblichen Phenolrots überprüft werden.

Wir berichten deshalb über die Ergebnisse dünnschichtchromatographischer Untersuchungen von Phenolsulfonphthaleinlösung der Fa. Merck, Darmstadt (Konzentration 0,6 und 6,0 g/l) und der Fa. Difco., Detroit.

\section{Material und Methoden}

Mit Hilfe eines Desaga-Streichgerätes wird auf mit Aceton gereinigte, fettfreie Glasplatten vom Format $20 \cdot 20 \mathrm{~cm}$ eine etwa $0,25 \mathrm{~mm}$ dünne Kieselgelsçhicht (Kiẹselgel nach Stahl der Fạ. Merck, Darmstadt, mit einer mittleren Korngtöße von $10-40 \mu \mathrm{m}$ ) aufgetragen.

Insgesamt werden 6 Proben auf der Startlinie des Chromatogramms, $20 \mathrm{~mm}$ vom Plattentand entfernt und mit einem seitlichen Abstand von etwa $20 \mathrm{~mm}$ mit Hilfe einer Mikroliterspritze auf einer Platte aufgetragen.

Es wurden die folgenden Proben untersucht:

1. Phenolsulfonphthalein der Fa. E. Merck, Darmstadt, in einer Konzentration von $6,0 \mathrm{~g} / \mathrm{l}$. Chargen Nr. J 2202606. Aufgetragen wurden $3 \mu \mathrm{l}$ der Originallösung.

2. Phenolsulfonphthaleinlösung zur Nierenfunktionsprüfung der Fa. E. Merck, Darmstadt. Die Originallösung enthält laut Pro- spekt $600 \mathrm{mg}$ Farbstoff im Liter. Sie wurde im Rotationsverdampfer bei $40^{\circ}$ auf ein Zehntel des Ausgangsvolumens eingeengt. Von dieser Lösung wurden ebenfalls $3 \mu \mathrm{l}$ zur Trennung auf die Platten aufgetragen.

3. Phenolsulfonphthalein als Trockensubtsanz der Fa. E. Merck, Darmstadt. Reagenzien Nr. 6163440. $300 \mathrm{mg}$ Trockensubstanz wurden mit etwa $30 \mathrm{ml}$ dest. Wasser aufgeschwemmt und zur Lösung mit $1 \mathrm{~N} \mathrm{NaOH}$ auf $\mathrm{pH} 7,1$ titriert. Mit dest. Wasser wurde auf $50 \mathrm{ml}$ aufgefüllt, so daß die Farbstoffkonzentration $6 \mathrm{~g} / 1$ betrug. Aufgetragen wurden Probenvolumina von $3 \mu \mathrm{l}$.

4. Zur Orientierung wurde in gleicher Weise Phenolsulfonphthalein der Fa. Difco Lab., Detroit, Mich., USA, Control No. 510210 als Trockensubstanz untersucht. Es wurde, wie für das Präparat von Merck angegeben, in Lösung gebracht und in Probenvolumina von $3 \mu \mathrm{l}$ auf die Platten gebracht.

Das Entwickeln des Chromatogramms erfolgte aufsteigend bei Raumtemperatur mit Butanol p. a.: Äthanol absolut. p. a.: 5 proz. $(v / v)$ Ammoniak = 4:1:1 (v/v).

Jede Mischung wurde nur für einen Lauf.verwendet. Die Trennkammer wurde zur Kammersättigung mit Fließpapier, das mit Fließmittel getränkt war, ausgeschlagen. Die Fließmittelhöhe im Trenngefä $\beta$ betrug etwa $1,3 \mathrm{~cm}$. Die Trennstrecke war in allen Versuchen $15 \mathrm{~cm}$ lang und wurde von der Fließmittelfront in etwa drei Stunden zurückgelegt.

Es wurden mit jeder Lösung jeweils drei Dünnschichtplatten ( $a, b$ und c) mit 6 Auftragsstellen beschickt, so daß zur Auswertung 18 Bahnen der gleichen Lösung zur Verfügung standen.

Die $R_{\mathrm{F}}$-Werte der bei Tageslicht sichtbaren und der bei Anregung mit UV-Licht fluoreszierenden Fraktionen wurden bestimmt und jeweils für die sechs Proben einer Platte und für die zusammengehörenden drei Platten ciner Serie gemittelt. Die Intensität der Flecken konnte durch Ansprühen mit 5proz. Ammoniaklösung teilweise verstärkt werden.

\section{Ergebnisse}

In Tabelle 1 sind die Ergebnisse der dünnschichtchromatographischen Trennungen der vier untersuchten Phenolrotlösungen aufgezeichnet.

Mit dem angewandten Trennverfahren waren mindestens fünf und maximal sieben verschiedene Fraktionen des Farbstoffs sicher nachweisbar. 
Tab. 1

Obersicht über die dünnschichtchromatographischen Trennergebnisse. $\overline{\mathbf{x}}_{1}$ : gemittelte $R_{\mathrm{F}}-$ Werte der Platten a-c $\overline{\mathbf{x}}_{2}$ : gemittelte $\overline{\mathbf{x}}_{1}$-Werte

\begin{tabular}{|c|c|c|c|c|c|c|c|c|c|}
\hline Testsubstanz & $\begin{array}{c}\text { Anzahl der } \\
\text { Flecken }\end{array}$ & & $\dot{\mathrm{x}}_{1}(\mathrm{n}=$ & & $\bar{x}_{2}(n=18)$ & Fleckenform & $\begin{array}{l}\text { Far } \\
\text { (Tages }\end{array}$ & $\begin{array}{l}\text { rbe } \\
\text { slịcht) }\end{array}$ & $\begin{array}{c}\text { Fluoreszenz } \\
\left(\lambda_{\text {Excit }}=366 \mathrm{~nm}\right)\end{array}$ \\
\hline $\begin{array}{l}\text { Phenolrot der Fa. Merck } \\
6,0 \text { g/l } \\
\text { Chargen Nr. J2202606 }\end{array}$ & 7 & $\begin{array}{c}\text { a. } \\
0,14 \\
0,16 \\
0,18 \\
0,42 \\
0,43 \\
0,52 \\
0,61\end{array}$ & $\begin{array}{c}\text { b. } \\
0,15 \\
0,16 \\
0,18 \\
0,41 \\
0,43 \\
0,52 \\
0,61\end{array}$ & $\begin{array}{c}c . \\
0,12 \\
0,15 \\
0,17 \\
0,40 \\
0,42 \\
0,52 \\
0,60\end{array}$ & $\begin{array}{l}0,14 \\
0,16 \\
0,18 \\
0,41 \\
0,43 \\
0,52 \\
0,61\end{array}$ & $\begin{array}{l}\text { halbmondförmig } \\
\text { halbmondförmig } \\
\text { halbmondförmig } \\
\text { längsoval } \\
\text { queroval } \\
\text { rund } \\
\text { rund }\end{array}$ & $\begin{array}{l}\text { rot } \\
\text { rot } \\
\text { gelb }\end{array}$ & - & $\begin{array}{l}\text { mattgelb } \\
\text { türkisblau } \\
\text { mattge-lb } \\
\text { gräulich-weiß }\end{array}$ \\
\hline $\begin{array}{l}\text { Phenolrot der Fa. Merck } \\
0,6 \text { g/1 } \\
\text { "zur Nierenfunktions- } \\
\text { prüfung" Originallösung auf } \\
\text { "/, des Volumens eingeengt }\end{array}$ & 6 & $\begin{array}{l}0,09 \\
0,19 \\
0,35 \\
0,36 \\
0,50 \\
0,61\end{array}$ & $\begin{array}{l}0,09 \\
0,18 \\
0,30 \\
0,32 \\
0,45 \\
0,58\end{array}$ & $\begin{array}{l}0,12 \\
0,20 \\
0,37 \\
0,40 \\
0,52 \\
0,62\end{array}$ & $\begin{array}{l}0,10 \\
0,19 \\
0,34 \\
0,36 \\
0,49 \\
0,60\end{array}$ & $\begin{array}{l}\text { halbmondförmig } \\
\text { halbmondförmig } \\
\text { rund } \\
\text { queroval } \\
\text { längsoval } \\
\text { längsoval }\end{array}$ & $\begin{array}{l}\text { brau } \\
\text { rot } \\
\text { gelbo }\end{array}$ & $\begin{array}{l}\text { n-rot } \\
\text { orange } \\
=\end{array}$ & $\begin{array}{l}\text { bläulich-weiß } \\
\text { mattgelb } \\
\text { grâulich-weiß }\end{array}$ \\
\hline $\begin{array}{l}\text { Phenolrot der Fa. Merck } \\
6,0 \text { g/l } \\
\text { Reagenzien Nr. } 6163440 \\
\text { Ansatz siehe Text }\end{array}$ & 6 & $\begin{array}{l}0,16 \\
0,22 \\
0,40 \\
0,51 \\
0,55 \\
0,61\end{array}$ & $\begin{array}{l}0,14 \\
0,22 \\
0,41 \\
0,50 \\
0,54 \\
0,60\end{array}$ & $\begin{array}{l}0,15 \\
0,22 \\
0,41 \\
0,50 \\
0,54 \\
0,60\end{array}$ & $\begin{array}{l}0,15 \\
0,22 \\
0,41 \\
0,50 \\
0,54 \\
0,60\end{array}$ & $\begin{array}{l}\text { halbmondförmig } \\
\text { halbmondförmig } \\
\text { rund } \\
\text { rund } \\
\text { rund } \\
\text { rund }\end{array}$ & $\begin{array}{l}\text { rot } \\
\text { gelb } \\
\text { gelb }\end{array}$ & & $\begin{array}{l}\text { hellgelb } \\
\text { mattgelb } \\
\text { weißgelb }\end{array}$ \\
\hline $\begin{array}{l}\text { Phenolrot der Fa. Difco } \\
6,0 \text { g/l } \\
\text { control No. } 510210 \\
\text { Ansatz siehe Text }\end{array}$ & 5 & $\begin{array}{l}0,17 \\
0,21 \\
0,43 \\
0,55 \\
0,60\end{array}$ & $\begin{array}{l}0,18 \\
0,23 \\
0,46 \\
0,58 \\
0,62\end{array}$ & $\begin{array}{l}0,17 \\
0,22 \\
0,44 \\
0,58 \\
0,62\end{array}$ & $\begin{array}{l}0,17 \\
0,22 \\
0,44 \\
0,57 \\
0,61\end{array}$ & $\begin{array}{l}\text { halbmondförmig } \\
\text { rund-rechteckig } \\
\text { rund } \\
\text { rund } \\
\text { rund }\end{array}$ & rot & $=$ & $\begin{array}{l}\text { weiß } \\
\text { grau-bläulich } \\
\text { mattgelb } \\
\text { mattgelb }\end{array}$ \\
\hline
\end{tabular}

Auffällig war die unterschiedliche Fleckenzahl bei den beiden geprüften Farbstofforiginallösungen der $\mathrm{Fa}$. E. Merck, Darmstadt. So wies die konzentrierte Phenolrotlösung (Untersuchungsmaterial Nr. 1) gegenüber der weniger konzentrierten (Untersuchungsmaterial Nr. 2) eine zusätzliche Fraktion auf. Die Flecken mit den $R_{\mathrm{F}}$-Werten $0,18-0,22$ waren am farbintensivsten nach Besprühen mit Ammoniaklösung.

\section{Diskussion}

Die dünnschichtchromatographischen Trennungen ergaben, daß keine der untersuchten Phenolrotlösungen als einheitliche Lösung zu bezeichnen ist. Die unterschiedliche Fleckenzahl bei den Farbstofflösungen weist auf den wechselnden Charakter der Verunreinigungen hin. Aufgrund dieser qualitativen Angaben über die Reinheit des Farbstoffs sollten beim Durchführen des Phenolrottestes in der klinischen Routinediagnostik folgende Bedenken Berücksichtigung finden:

1. Beigemischte Fremdprodukte mit unbekannter Ausscheidungskinetik könnten für das Phenolrotdefizit in Bilanzversuchen verantwortlich sein.
2. Es bestehen toxikologische Bedenken gegen die Applikation einer nicht streng definierten Substanz beim Menschen. Phenolrot galt bisher als nicht toxisch und gut verträglich. Wie durch ein Rundschreiben der Firma E. Merck, Darmstadt, vom 7.6.1971 bekannt wurde, sind jedoch allergische und anaphylaktische Reaktionen nach Phenolrotapplikation möglich. Es wird in diesem Schreiben auch von einem Todesfall berichtet (6).

3. Wenn, wie es manche Autoren empfehlen (4), sehr große Mengen des Farbstoffs injiziert werden, die die bisher üblichen Dosen von $6 \mathrm{mg}$ Phenolrot pro Test bis um das 100 fache überschreiten, sollte die Toxizität des Phenolrots und seiner Verunreinigungen erneut überprüft werden.

Für die Phenolrotbestimmung im Serum haben wir eine verbesserte Methode angegeben (7).

Normalwerte für diese Methode können jedoch erst dann ermittelt werden, wenn ein Farbstoff zur Verfügung steht, der sich auch bei dünnschichtchromatographischer Untersuchung als einheitlich erweist.

Wir danken Frau Loth und Frau Beinhauer für ihre sorgfältige Mitarbeit.

\section{Literatur}

1. Rowntree, L. G. und J. T. Geraghty, J. Pharmacol. exper. Therap., Baltimore 1, 579 (1910). - 2. OpfermanN, G., Der intravenöse Phenolrottest. Inauguraldissertation, Freie Universität Berlin (1968). - 3. HöfFLER, D., G. OfFERMANN und H. Frenz, Dtsch. med. Wschr. 93, 397 (1968). - 4. SchWARTZKOPFF, W., Z. exper. Med. 150, 120 (1969). - 5. McLeOd, G. M., A. B.
French, C. J. Good und F. S. Wright, J. Laborat. Clin. Med., S. Louis 71, 192 (1968). - 6. Rundschreiben der Fa. E. Merck, 61 Darmstadt, Postfach 4119, Frankfurter Straße 250, vom 7.6. 1971, gez. von Dr. Bayer und Dr. Iltgen. Betr.: Todesfall nach Injektion von Phenolsulfonphthalein. - 7. FABricrus, W. und B. Marowskr, diese Z. 8, 431 (1970).
Dr. Bernhard Marowski 1 Berlin 12 Windscheidstr. 12
Dr. Wolfgang Fabricius

Siemens Aktiengesellschaft, Vertrieb Datentechnik 1 Berlin 61, Schöneberger Str. $2-4$ 